\title{
H19DMR methylation analysis in patients with Beckwith-Wiedemann syndrome and isolated hemihyperplasia
}

\author{
Marcus Vinícius de Matos Gomes, Sílvio Avelino dos Santos and Ester Silveira Ramos \\ Universidade de São Paulo, Faculdade de Medicina de Ribeirão Preto, Departamento de Genética, \\ Ribeirão Preto, São Paulo, Brazil.
}

\begin{abstract}
Beckwith-Wiedemann syndrome (BWS) is a congenital overgrowth disorder of complex and heterogeneous etiology involving alterations in genomic imprinting. The cause of isolated hemihyperplasia $(\mathrm{IHH})$ is unknown but might be due to partial or incomplete expression of BWS because both these conditions share predisposition for the same types of neoplasias. We investigated the methylation pattern of the putative imprinting control region H19DMR using peripheral blood from 12 patients, six with clinical features of BWS and six with $\mathrm{IHH}$. All the patients had normal karyotypes and paternal uniparental disomy (UPD) was excluded in 10 informative cases. The normal H19DMR methylation pattern was found in eight informative patients, indicating that H19DMR methylation was not related to their condition. We suggest that the absence of neoplasias in the BWS and IHH patients studied might be related to the absence of UPD and to the presence of normal H19DMR methylation.
\end{abstract}

Key words: Beckwith-Wiedemann syndrome, isolated hemihyperplasia, genomic imprinting, DNA methylation, uniparental disomy, H19DMR.

Received: April 13, 2004; Accepted: November 23, 2004.

Beckwith-Wiedemann syndrome (BWS) is a congenital disorder characterized by overgrowth and predisposition to some types of cancer. The most common features associated with BWS are increased growth, macroglossy, ear lobe creases and/or posterior helical indentations, and abdominal wall defects (Elliott et al., 1994). Children with BWS have a 7-21\% risk of developing embryonic malignancies, most notably Wilms' tumor of the kidney (Weksberg et al., 2001). Hemihyperplasia or asymmetric growth of one or more parts of the body can be observed in approximately $12.5 \%$ of individuals with BWS and is present in $40 \%$ of BWS patients who develop tumors (Wiedemann, 1983). It has been suggested that isolated hemihyperplasia $(\mathrm{IHH})$ represent partial or incomplete expression of BWS because both these conditions produce a predisposition for the same types of neoplasias (SoteloAvila et al., 1980).

The etiology of BWS is both complex and heterogeneous but is characterized by genetic and epigenetic alterations in the $11 \mathrm{p} 15.5$ chromosomal region which contains two genetic domains (telomeric and centromeric) regulated

Send correspondence to Marcus Vinícius de Matos Gomes. Universidade de São Paulo, Faculdade de Medicina de Ribeirão Preto, Departamento de Genética, Avenida Bandeirantes 3900, 14049900 Ribeirão Preto, São Paulo, Brazil. E-mail: mvmgomes@ genbov.fmrp.usp.br. by genomic imprinting, a process that leads to the silencing of a specific parental allele. The $I G F 2$ and $H 19$ genes have been mapped to the telomeric domain, while the CDKN1C (also known as p57Kip2), KVLQT1 and LIT1 (also known as $K v L Q T 1 A S$ ) genes map to the centromeric domain (Maher and Reik, 2000). It has been suggested that two imprinting control regions (ICR), H19DMR and KvDMR, control the expression of these genes. Alteration of the H19DMR methylation pattern has been associated with loss of imprinting. of the IGF2 gene (Bell and Felsenfeld 2000), while abnormal methylation of KvDMR has been associated with loss of imprinting of the LIT1 and CDKN1C genes (Diaz-Meyer et al., 2003).

Paternal uniparental disomy (UPD) associated with BWS results in over-expression of growth promoter genes, which are normally activated only in the paternal chromosome (Kotzot, 1999). Catchpoole et al. (1997) reported that UPD in the $11 \mathrm{p} 15.5$ region occurred in about $20 \%$ of sporadic BWS cases.

The etiology of IHH still remains unclear with most reported cases having been sporadic and without chromosomal rearrangements, although it has been suggested that IHH represents patchy over-expression of the IGF2 gene due to defective imprinting (Hoyme et al., 1998). The frequency of hemihyperplasia among BWS patients with uniparental disomy or aberrant methylation of both 
H19DMR and KvDMR has been found to be significantly higher than that in patients without such defects (DeBaun et al., 2002).

Loss of imprinting of the $I G F 2$ gene leading to expression of the normally silent maternal gene seems to be an important factor in the association between neoplasias and BWS and/or IHH. The abnormal H19DMR methylation observed in some patients with BWS may be related to a predisposition to develop tumors, especially Wilms' tumor (DeBaun et al., 2002). Loss of imprinting of H19DMR has also been shown in patients with hepatoblastomas, rhabdomyosarcomas, and gonadoblastomas (Bliek et al., 2001; Weksberg et al., 2001).

Knowledge of the etiologic mechanisms of BWS and IHH may be useful for patient management and for better genetic counseling of their families, because of which we undertook a study to identify alterations leading to constitutional loss of imprinting in BWS and IHH patients. In this study we took blood samples from BWS and IHH patients and investigated them for chromosomal rearrangements, paternal UPD and the methylation pattern of the putative imprinting control region H19DMR.

Molecular and cytogenetic analyses were carried out on two females and four males with BWS (median age $=9$ years) and three females and three males with IHH (median age $=10$ years), all being outpatients at the Ribeirão Preto School of Medicine, University of São Paulo, Ribeirão Preto, São Paulo, Brazil. The study was approved by the National Ethical Committee (CONEP) and informed consent was obtained from the participating families. Clinical diagnosis of BWS was based on three major features (macroglossia, pre or postnatal growth $>90$ th percentile, and abdominal wall defects) or two of these major features plus at least three minor features such as ear lobe creases or posterior helical ear pits, facial nevus flammeus, hypoglycemia, nephromegaly or hemihyperplasia (Elliott et al., 1994). All the BWS patients showed asymmetrical growth and were apparently sporadic cases. We included in the study IHH patients who showed asymmetrical overgrowth of one limb or one side of the body (with or without facial, trunk or visceral involvement) but excluded patients with neuromuscular alterations characteristic of other disorders.

Cytogenetic analysis was performed on peripheral blood lymphocytes after GTG-banding (Scheres, 1972) and genomic DNA was obtained from the same blood samples (Olerup e Zetterquist, 1992). All patients were first screened for paternal UPD by the restriction fragment length polymorphism (RFLP) method using the H19/RsaI (Zhang et al., 1992) and IGF2/ApaI (Tadokoro et al., 1991) polymorphisms and by D11S4177 and D11S922 microsatellite analysis (http:/www.ncbi.nlm.nch.gov, nucleotide access numbers Z53859 and Z16988).

The allele specific methylation pattern was evaluated by the differential methylation of HhaI RFLP mapped to the H19DMR region after digestion by the methylationsensitive HpaII restriction enzyme and polymerase chain reaction (PCR) amplification (Jinno et al.,1996). In the presence of normal H19DMR mono-allelic methylation HpaII digests only the unmethylated allele preventing its amplification but when bi-allelic methylation (hypermethylation) is present both alleles remain undigested and are amplified by the PCR. In non-informative cases (HhaI homozygotes) we also analyzed AvaI RFLP. Both HhaI or AvaI digestion was carried out on DNA samples previously digested with HpaII and on samples with no HpaII digestion. For the HhaI polymorphism the PCR was performed using the primers H1 (5, CAATGAGGTGTCCCAGTT CCA 3') and H2 (5' CACATAAGTAGGCGTGACTTGA $3^{\prime}$ ') and for the AvaI alleles nested PCR was performed using the primers V1 (5' GAGCCTGCCAAGCAGAGCG 3') and V2 (5' CACATAAGTAGGCGTGACTTGA 3') and the internal primers N1 (5' GTGTCCCCATTCTTT GGATG 3') and N2 (5' GTTTCACACTAGGGCCGAGA $\left.3^{\prime}\right)$. Alleles were visualized using electrophoresis on $2 \%$ agarose gel and ethidium bromide staining.

None of the 12 patients studied had chromosomal alterations. The presence of paternal UPD was excluded in three patients based on analysis of the H19/RsaI polymorphism and the presence of a maternally inherited allele, the remaining cases being non-informative. Genotyping of the D11S4177 and D11S922 loci enabled the identification of bi-parental inheritance in 10 patients (Figure 1, Table 1). Two BWS patients were non-informative in both analyses.

Analysis of H19DMR methylation revealed a normal pattern in eight patients, $5 / 6$ patients with $\mathrm{IHH}$ and $3 / 6$ with BWS (Figure 2), the four remaining cases being noninformative due to RFLP homozygosity. A summary of the results is shown in Table 1.

The absence of chromosomal abnormalities in the BWS and IHH patients analyzed agrees with previous reports showing a low frequency (1-2\%) of chromosome aberrations in BWS cases (Maher and Reik, 2000).

Paternal UPD occurs in approximately $20 \%$ of BWS cases as a result of meiotic and/or mitotic non-disjunctions (Catchpoole et al., 1997). Although Itoh et al. (2000) reported UPD mosaicism in BWS and observed that the most severely affected organs presented the highest percentage
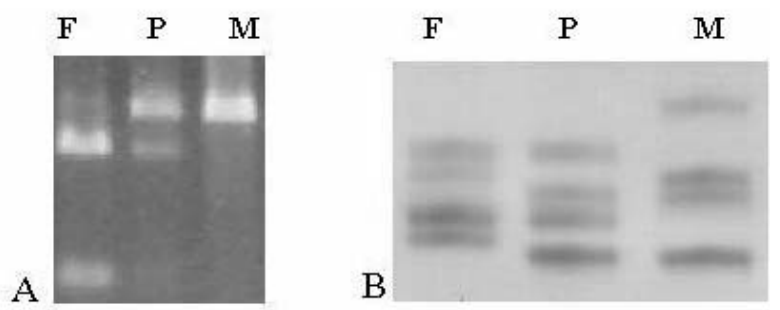

Figure 1 - Exclusion of uniparental disomy by comparison of patient and parental genotypes. Inheritance of maternal and paternal alleles as assessed by (A) H19/RsaI RFLP and (B) microsatellite D11S4177 loci ( $\mathrm{F}=$ father, $\mathrm{M}=$ mother, $\mathrm{P}=$ patient $)$. 
Table 1 - Summary of the investigation of uniparental disomy (UPD) and H19DMR methylation pattern in patients with Beckwith-Wiedemann syndrome (BWS) and Isolated Hemihyperplasia (IHH).

\begin{tabular}{|c|c|c|c|c|c|c|}
\hline \multirow[b]{2}{*}{ Patient } & \multicolumn{4}{|c|}{ UPD } & \multicolumn{2}{|c|}{ Methylation pattern } \\
\hline & H19/RsaI & $I G F 2 / A p a I$ & D11S4177 & D11S922 & DMR/HhaI & $\mathrm{DMR} / A v a I$ \\
\hline \multicolumn{7}{|c|}{ BWS } \\
\hline 1 & NI & NI & NI & NI & NI & NI \\
\hline 2 & NI & NI & NI & NI & NI & NI \\
\hline 3 & $\mathrm{BP}$ & NI & $\mathrm{BP}$ & $\mathrm{BP}$ & MAM & NA \\
\hline 4 & NI & NI & $\mathrm{BP}$ & $\mathrm{BP}$ & MAM & NA \\
\hline 5 & NI & NI & $\mathrm{BP}$ & $\mathrm{BP}$ & NI & NI \\
\hline 6 & NI & $\mathrm{NI}$ & $\mathrm{BP}$ & BP & $\mathrm{NI}$ & MAM \\
\hline \multicolumn{7}{|c|}{$\mathrm{IHH}$} \\
\hline 7 & NI & NI & $\mathrm{BP}$ & BP & MAM & NA \\
\hline 8 & BP & NI & BP & NI & MAM & NA \\
\hline 9 & NI & NI & $\mathrm{BP}$ & NI & NI & NI \\
\hline 10 & NI & NI & $\mathrm{BP}$ & NI & MAM & NA \\
\hline 11 & BP & $\mathrm{NI}$ & BP & BP & MAM & NA \\
\hline 12 & NI & NI & BP & BP & MAM & NA \\
\hline
\end{tabular}

$\mathrm{NI}=$ non-informative, $\mathrm{BP}=$ bi-parental inheritance; $\mathrm{MAM}=$ mono-allelic methylation; $\mathrm{NA}=$ not analyzed .

of cells with UPD, Gaston et al. (2001) demonstrated that when UPD is present in tongue and tumor samples it was also detectable in peripheral blood and was always associated with abnormal bi-allelic methylation in the H19DMR region. In our study, the absence of UPD in 10 patients agreed with the H19DMR mono-allelic methylation seen in the eight informative patients.

It has been postulated that $I G F 2$ over-expression caused by loss of imprinting is one of the main factors responsible for overgrowth in BWS and IHH patients (Weksberg et al., 1993). We could not analyze IGF2 expression because our patients were not heterozygous for IGF2/ApaI RFLP (Table 1), which precluded reverse transcription (RT) expression studies using RT-PCR). Because of this we opted to carry out 'indirect' expression analysis based on methylation of the putative H19DMR imprinting
A

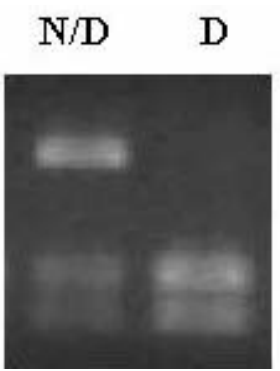

$\mathrm{N} / \mathrm{D}$

B

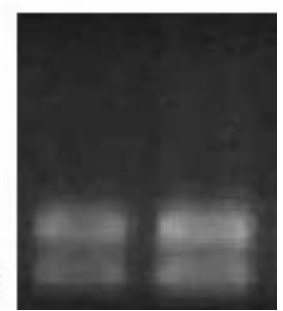

Figure 2 - The H19DMR methylation pattern as determined by HhaI RFLP analysis (Lane N/D = HpaII undigested DNA; Lane D = HpaII digested DNA): A) Monoallelic methylation demonstrated by the absence of the larger HhaI allele (upper band) in the HpaII digested DNA; B) Non-informative case homozygous for the smaller HhaI allele (see text for details). control region. Sporadic BWS cases with bi-allelic $I G F 2$ expression and bi-allelic H19DMR methylation have been reported by Reik et al. (1995) but in our series of patients the eight informative cases showed a normal H19DMR methylation pattern, although these patients might have alterations in the KvDMR imprinting control region as reported by Brown et al. (1996) in BWS patients.

Regarding predisposition to tumors, it has been postulated that there is a relationship between cancer risk and loss of IGF2 imprinting and/or abnormal methylation of H19DMR because both effects have been described in neoplasias (principally Wilms' tumor) in children with BWS (Bliek et al., 2001). DeBaun et al. (2002) hypothesized that abnormal methylation in the telomeric domain (H19DMR) may be associated with overgrowth and predisposition to tumors, while abnormal methylation in the centromeric domain may be associated with macrosomy and abdominal wall defects but not with predisposition to tumors. Our patients were all at least five years old at the time of our study and have been followed up and screened for tumors at least twice a year by ultrasound, none of them having developed neoplastic processes. In spite of the small sample size, we suggest that the absence of neoplasias (especially Wilms' tumor) in the BWS and IHH patients examined in our study may be related to the absence of UPD and the presence of normal H19DMR methylation.

\section{Acknowledgments}

We thank the patients' families who spontaneously participated in this study, and Dr. Cláudia A. Rainho for intellectual and technical assistance. This work was sup- 
ported by grants from the Brazilian agencies $\mathrm{CNPq}$, FAEPA, FAPESP, and PRONEX.

\section{References}

Bell AC and Felsenfeld G (2000) Methylation of a CTCFdependent boundary controls imprinted expression of the Igf2 gene. Nature 405:482-485.

Bliek J, Maas SM, Ruijter JM, Hennekan RC, Alders M, Westerveld A and Mannens MM (2001) Increased tumour risk for BWS patients correlates with aberrant $\mathrm{H} 19$ and not KCNQ1OT1 methylation: Occurrence of KCNQ1OT1 hypomethylation in familial cases of BWS. Hum Mol Genet 10:467-476.

Brown KW, Villar AJ, Bickmore W, Clayton-Smith J, Catchpoole D, Maher ER and Reik W (1996) Imprinting mutation in the Beckwith-Wiedemann syndrome leads to biallelic $I G F 2$ expression through an H19-independent pathway. Hum Mol Genet 5:2027-2032

Catchpoole D, Lam WW, Valler D, Temple IK, Joyce JA, Reik W, Schofield PN and Maher ER (1997) Epigenetic modification and uniparental inheritance of $H 19$ in Beckwith-Wiedemann syndrome. J Med Genet 34:353-359.

DeBaun MR, Niemitz EL, McNeil DE, Brandenburg SA, Lee MP and Feinberg AP (2002) Epigenetic alterations of H19 and LIT1 distinguish patients with Beckwith-Wiedemann syndrome with cancer and birth defects. Am J Hum Genet 70:604-611.

Diaz-Meyer N, Day CD, Kathod K, Maher ER, Cooper W, Reik W, Juncem C, Graham G, Algar E, Der Kaloustian VM and Higgings MJ (2003) Silencing of CDKNIC (p57KIP2) is associated with hypomethylation at KvDMR1 in BeckwithWiedemann syndrome. J Med Genet 40:797-801.

Elliott M, Bayly R, Cole T, Temple IK and Maher ER (1994) Clinical features and natural history of Beckwith-Wiedemann syndrome: Presentation of 74 new cases. Clin Genet 46:168-174.

Hoyme HE, Seaver LH, Jones KL, Procopio F, Crooks W and Feingold M (1998) Isolated hemihyperplasia (hemihypertrophy): Report of a prospective multicenter study of the incidence of neoplasia and review. Am J Med Genet 79:274-278.

Gaston V, Le Bouc Y, Soupre V, Burglen L, Donadieu J, Oro H, Audry G, Vazquez MP and Gicquel C (2001) Analysis of the methylation status of the KCNQ1OT and H19 genes in leukocyte DNA for the diagnosis and prognosis of BeckwithWiedemann syndrome. Eur J Hum Genet 9:409-418.

Itoh N, Becroft DM, Reeve AE and Morison IM (2000) Proportion of cells with paternal $11 \mathrm{p} 15$ uniparental disomy corre- lates with organ enlargement in Wiedemann-beckwith syndrome. Am J Med Genet 92:111-116.

Jinno $\mathrm{Y}$, Sengoku K, Nakao M, Tamate $\mathrm{K}$, Miyamoto $\mathrm{T}$, Matsuzaka T, Sutcliffe JS, Anan T, Takuma N, Nishiwaki K, Ikeda Y, Ishimaru T, Ishikawa M and Niikawa N (1996) Hum Mol Genet 5:1155-1161.

Kotzot D (1999) Abnormal phenotypes in uniparental disomy (UPD): Fundamental aspects and a critical review with bibliography of UPD other than 15. Am J Med Genet 82:265274.

Maher ER and Reik W (2000) Beckwith-Wiedemann syndrome: Imprinting in cluster revisited. J Clin Invest 105:247-252.

Olerup O and Zetterquist H (1992) HLA-DR typing by PCR amplification with sequence-specific primers (PCR-SSP) in 2 hours: An alternative to serological DR typing in clinical practice including donor-recipient matching in cadaveric transplantation. Tissue Antigens 39:225-235.

Reik W, Brown KW, Schneid H, Le Bouc Y, Bickmore W and Maher ER (1995) Imprinting mutations in the BeckwithWiedemann syndrome suggested by altered imprinting pattern in the IGF2-H19 domain. Hum Mol Genet 4:23792385.

Scheres JM (1972) Human chromosome banding. Lancet 1:849.

Sotelo-Avila C, Gonzalez-Crussi F and Fowler JW (1980) Complete and incomplete forms of Beckwith-Wiedemann syndrome: Their oncogenic potential. J Pediat 96:47-50.

Tadokoro K, Fuji H, Inoue T and Yamada M (1991) Polymerase chain reaction (PCR) for detection of Apal polymorphism at the insulin like growth factor II gene (IGF2). Nucl Acids Res 19:6967.

Weksberg R, Teshima I, Williams BR, Greenberg CR, Pueschel SM, Chernos JE, Fowlow SB, Hoyme E, Anderson IJ and Whiteman DA (1993) Molecular characterization of cytogenetic alterations associated with the BeckwithWiedemann syndrome (BWS) phenotype refines the localization and suggests the gene for BWS is imprinted. Hum Mol Genet 2:549-556.

Weksberg R, Nishikawa J, Caluseriu O, Fei YL, Wei C, Steele L, Cameron J, Smith A, Ambus I, Li M, Ray PN, Sadowski P and Squire J (2001) Tumor development in the BeckwithWiedemann syndrome is associated with a variety of constitutional molecular $11 \mathrm{p} 15$ alterations including imprinting defects of KCNQ1OT1. Hum Mol Genet 10:2989-3000.

Wiedemann HR (1983) Tumors and hemihypertrophy associated with Wiedemann-Beckwith syndrome. Euro J Pediat 141:29.

Zhang Y and Tycko B (1992) Monoallelic expression of the human $H 19$ gene. Nat Genet 1:40-44.

Associate Editor: Mayana Zatz 